\title{
LEFT THORACIC KIDNEY: A RARE FINDING AT INTRAVENOUS UROGRAPHY
}

\author{
Y. B. MENSAH and C. FORSON \\ Department of Radiology, Korle Bu Teaching Hospital, P. O. Box KB 77, Korle Bu, Accra, Ghana
}

Corresponding Author: Dr Yaw B Mensah

Email: ybmensah@yahoo.com

Conflict of Interest: None declared

\section{SUMMARY}

Thoracic kidney is a rare type of renal ectopia. Patients with thoracic kidneys are usually asymptomatic and the condition is usually discovered incidentally during radiological evaluation for other conditions or during thoracic surgery. An intravenous urography done for a thirty-eight year old man referred on account of a seemingly small right kidney on an abdominal ultrasound scan, showed a normal right kidney and a left thoracic kidney. Thoracic kidney is a rare but an important cause of a thoracic 'mass' or 'elevated hemi diaphragm'. It should be considered in the evaluation of such patients to prevent unnecessary surgical interventions and image guided biopsies.

Key Words: Thoracic kidney, Ectopic kidney, intravenous urography.

\section{INTRODUCTION:}

Thoracic kidney is a rare type of renal ectopia. ${ }^{1,2}$ It accounts for less than $5 \%$ of renal ectopia. ${ }^{2,3}$ Most cases are asymptomatic and discovered as incidental finding during the evaluation of a suspected mass on chest radiography or at thoracotomy. ${ }^{1,3}$ Intravenous Urography(IVU), ultra sonography, computed tomography (CT) or magnetic resonance imaging (MRI) enable differentiation from other juxtadiaphragmatic masses. ${ }^{1,4}$ This condition shows a slight left-sided preponderance.

It is commoner in males than females $(2: 1) .^{2,3,5,6}$ Renal ectopy in general is a relatively common entity ( 1 in 900 patients). The ectopic kidney may be pelvic, iliac, abdominal, thoracic, contra lateral, or crossed. ${ }^{1,7}$ In embryogenesis, the kidneys are formed early in the fifth gestational week. Initially located in the pelvis, they migrate cranially such that by the third month they are at the level of L2 vertebra and meet the suprarenal glands. In the process, they may become arrested at any site above their original point in the pelvis.
In certain situations however, the ascending developing kidney may 'over-shoot' and ascend to a higher location than normal, resulting in thoracic ectopia. This is believed to be due to delayed closure of the diaphragm or accelerated ascent of the kidney.,3,5,6 Not much information on thoracic kidney is available in our part of the world. We are therefore presenting this case to add to the knowledge already available in our part of the world.

\section{CASE REPORT}

A 38 year old man was referred from a polyclinic in Accra to the Department of Radiology, Korle Bu Teaching Hospital for an intravenous urogram (IVU) to assess the function of his right kidney. This was because he had had an abdominal ultrasound scan done earlier for abdominal pain which said his right kidney was small. No mention was made of the left kidney in the request. The patient did not have any history of trauma or surgery. The IVU showed a normal right kidney with adequate function. The left kidney was seen in the left thoracic cavity. The calyces of the left kidney were full, the pelvis was normal and the ureter was elongated but inserted orthotopically in the bladder. The ureter exited the thorax through the diaphragm (Figure 1).

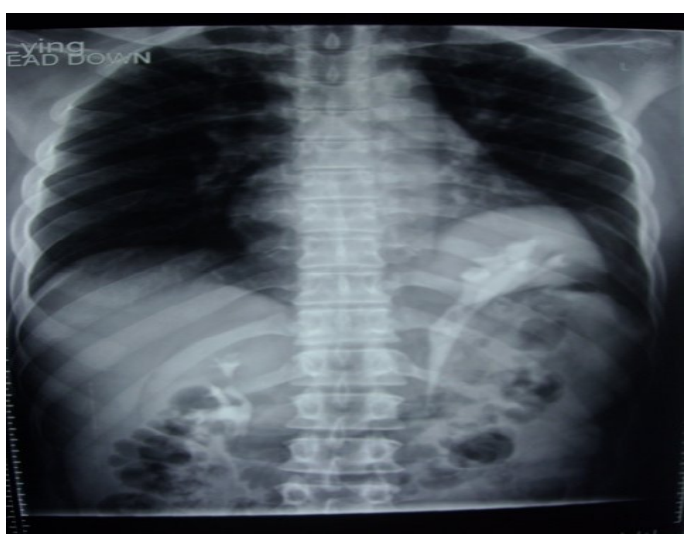

Figure 1 IVU with thoracic kidney 
Based on the IVU findings, chest and abdominal CT scans were done to assess the left kidney, renal vessels and suprarenal gland. These studies showed a malrotated left kidney in the postero-inferior aspect of the left hemi-thorax. It had a normal size and function (Figures 2 and 3$)$.

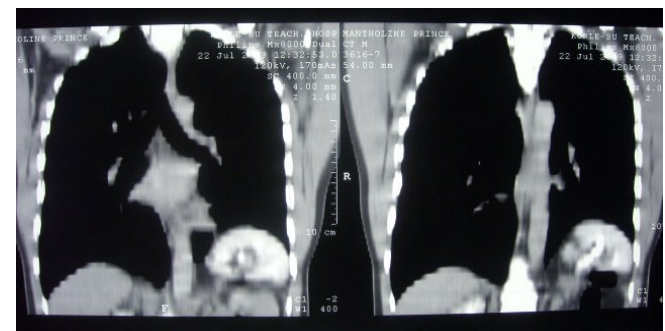

Figure 2 CT scan with coronal reconstruction showing left thoracic kidney

The left ureter exited the hemi-thorax through diaphragm. The left renal arteries arose from the normal level and extended into the hemi-thorax where the kidney was located. The suprarenal gland was also at its normal location.

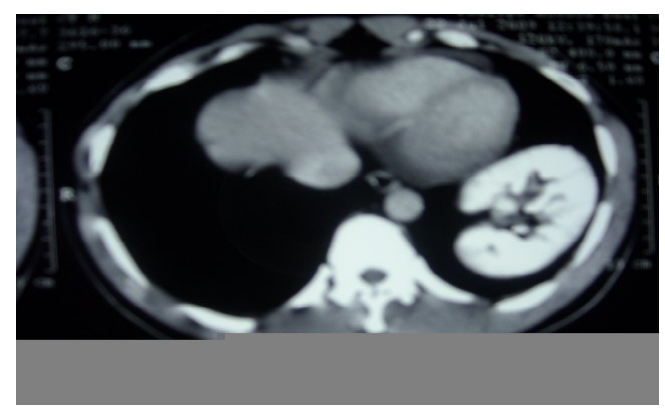

Figure 3 Axial CT scan showing a left thoracic kidney

\section{DISCUSSION}

This is the only reported case of a thoracic kidney at intravenous urography in last ten years in the Department of Radiology, Korle Bu Teaching hospital which conducts an average of 750 intravenous urographies a year. Not many cases have been reported in the world either. Between 1940 and 2003 only 200 cases had been reported in the world. ${ }^{5}$ The condition may be developmental or develop as a result of previous trauma. Our case is likely to be the developmental type since there has been no history of trauma or surgery. Patients are usually asymptomatic individuals who lead a normal life. This condition is most often diagnosed during a diagnostic work up for other problems. ${ }^{1,3,5}$ Our patient for instance was sent to our unit because a sonographer thought the right kidney was small. As was the case in our patient, usually both the thoracic kidney and the contralateral kidney in patients with a thoracic kidney are normal: associated anomalies in other organ systems are extremely rare and not consistent. ${ }^{3}$ The incidence of complications such as calculi or infection is not increased unlike other types of renal ectopia. ${ }^{2,5}$ The ipsilateral ureter is usually elongated and not ectopic.

In most cases the suprarenal glands, spleen and renal vessels are normally situated, which was the case in our patient. ${ }^{3,5} \mathrm{~A}$ thoracic kidney is an asymptomatic condition. A correct diagnosis of this condition would save patients from undergoing unnecessary surgical interventions and image guided biopsies. It must therefore be borne in mind in the evaluation of patients with suspected elevated hemi diaphragm or masses in the inferior aspect of the thorax. This information should also be available to the patients to enable him or her alert his or her doctor anytime any intervention is contemplated.

\section{ACKNOWLEDGEMENT}

We are grateful to Mr. Barnor, Mr. Benard Botwe and Mrs. Jennifer Tay of Radiology Department, Korle Bu Teaching Hospital for the role they played in the acquisition of the images. We are also grateful to Dr. Kwadwo Mensah for editing the document.

\section{REFERENCES}

1. Yalcinbas Y. K, Sasmaz H, Canbaz S. Thoracic left kidney: a differential diagnostic dilemma for thoracic surgeons. Ann Thorac Surg 2001;72:281283

2. Demirpolat G, Guney B, Savas R, Alper H. Thoracic Kidney Associated with Partially Intrathoracic Spleen: CT and MRI Findings. Clinical Radiology Extra. 2003; 58: 10-12

3. Lai C, Chiang W, Yang J, Yan H, Lin S, Chen Y, Wu K, Hsieh B. Thoracic kidney and contralateral ureteral duplication-a case report and review of the literature. Nephrology Dialysis Transplantation 2006 21(3):799-801.

4. Bugden W. F. Two Cases of Intra-Thoracic Kidney. Dis Chest. 1950;17; 357-359

5. Srinivasan V., Balasubramani N., Hariprasad B., Krishna Rao U., Thanasekaraan V.. Indian J Chest Dis Allied Sci 2003; 45:55-57.

6. Madani A, Ghassemi K, Ataei N, Mehdizadeh N, Esfehani ST, Mohseni P, Barkhordari H, Hadadi M. Thoracic Ectopic Kidney with Diaphragmatic Hernia. Tanaffos 2006; 5(2): 69-72

7. Clarkson L. M, Potter S. An unusual thoracic mass. British Journal of Radiology. 2009;82: 27 28. 